P\&A Año 2, N.2

enero-junio de 2017

pp. 29-39

\title{
Resumen
}

Durante la década del 60, la ausencia de una escuela de Arquitectura en Arequipa llevó a un grupo de jóvenes peruanos a estudiar en diferentes universidades argentinas. A mediados de la misma década se fundó en Arequipa una escuela de Arquitectura, con algunos profesores extranjeros, tres de los cuales fueron encargados de reorganizar la universidad bajo los nuevos criterios pedagógicos propuestos en el Plan Chaco. Al retornar, los jóvenes que habían partido a formarse en Argentina, conformaron la vanguardia académica arequipeña, aplicaron criterios pedagógicos y metodologías que aún no se usaban en Perú, poniéndolos en práctica para reorganizar la nueva escuela de Arequipa, con lo cual obtuvieron resultados positivos que la diferenciaron de las otras universidades de Arquitectura del país.

Palabras clave: Conexiones académicas, vanguardia académica arequipeña, metodologías, criterios pedagógicos, Argentina, Arequipa

\section{La vanguardia académica arequipeña. Conexiones entre arquitectos peruanos y argentinos. Periodo 1964-1987*}

\author{
The academic vanguard of Arequipa. Connections between Peruvian and Argentinian \\ architects. 1964-1987 period
}

\author{
Arq. María Mercedes Tapia Sasot**
}

Recibido: 10 de abril de 2017

Aceptado: 5 de junio de 2017

\begin{abstract}
The absence of a School of Architecture in Arequipa during the 1960s led a group of young Peruvians to study at different Argentinian universities. In the mid-1960s was a school of Architecture founded in Arequipa, with some foreign professors, three of them were placed in charge of the reorganization of the university under the new pedagogical principles that were proposed in the so-called Plan Chaco. The young Peruvian students returned to their hometown, and participated in what is known as the academic vanguard of Arequipa. They applied pedagogical principles, and methodologies not yet used in Peru, reorganize the new Architecture School of Arequipa. Thanks to such implementations, they obtained positive results that drew a distinction between them and the difference with the other Architecture universities of the country.
\end{abstract}

Keywords: Academic connections, academic vanguard of Arequipa, methodologies, pedagogical principles, Argentina, Arequipa

\footnotetext{
* El presente artículo es el resultado de la interpretación de entrevistas y de la documentación recopilada por la autora para el desarrollo de la tesis de la maestría en Historia, Teoría y Crítica de la Arquitectura, de la Unidad de Posgrado de la Facultad de Arquitectura de la Universidad Nacional de Ingeniería. Es una investigación autofinanciada. Se declara no tener conflicto de intereses en la publicación de este artículo.

** Arquitecta egresada de la Universidad Nacional de Buenos Aires, Argentina. Actualmente se desempeña como docente en la Universidad de Ciencias Aplicadas, Perú.
} 
En el año 1974 se realizó en Huancayo un encuentro de facultades de Arquitectura del Perú, en el que convergieron estudiantes de prácticamente todas las existentes en el Perú. Entre los participantes hubo consenso respecto de la valorización de los resultados de la intervención de la delegación de la Facultad de Arquitectura de la Universidad Nacional San Agustín de Arequipa (UNSA), que se destacó por plantear un nuevo enfoque sobre la discusión de proyectos, metodologías y planes de estudio de las facultades de Arquitectura y Urbanismo del Perú.

La contribución al debate fue descollante, pues propusieron de manera consistente una experiencia y un enfoque basado en nuevos criterios pedagógicos y conceptos doctrinarios, confrontándose al resto de las facultades del Perú, particularmente a las de Lima (Universidad Nacional de Ingeniería, Universidad Ricardo Palma y Universidad Nacional Fede-

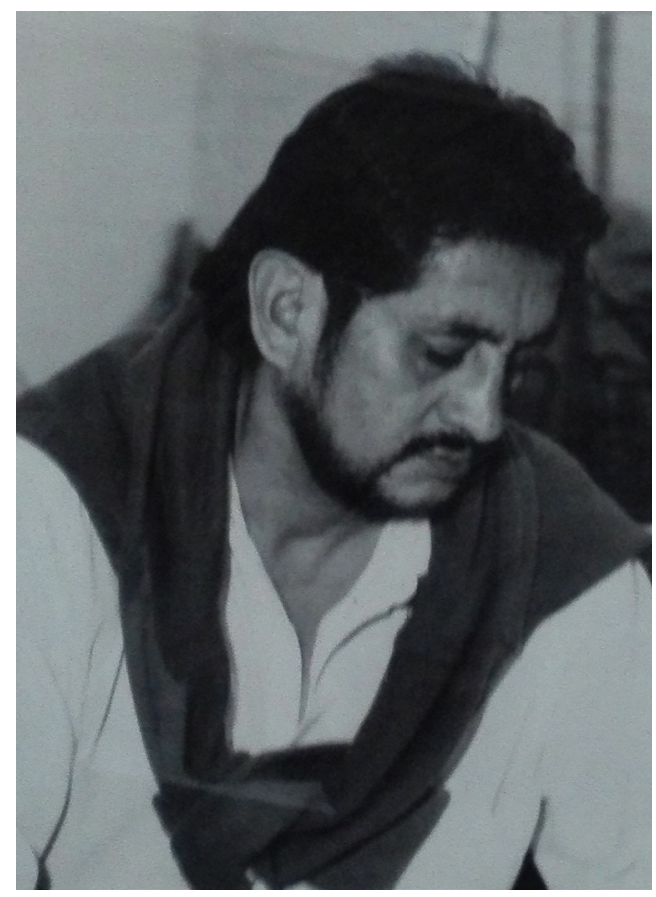

Figura 1. Arq. Edgardo Ramírez. Archivo Silvia Correa Viuda de Ramírez, s/f. rico Villarreal), que sugerían proyectos sin definiciones contundentes.

La delegación de Arequipa, encabezada por los arquitectos Edgardo Ramírez y Álvaro Pastor, entre otros, promovió las líneas de una participación coherente, convincente y crítica al sistema de enseñanza imperante. La participación arequipeña no fue un acto espontáneo y coyuntural, sino que estaba respaldada por una experiencia de cambios en las formas de ver y enseñar la arquitectura proveniente de Argentina. En Arequipa, estos cambios se produjeron a fines de los años 60 , antes que en las facultades de Lima, donde tuvieron lugar a mediados de los 70 .

Los arquitectos arequipeños, todos jóvenes docentes, habían sido los actores principales de los primeros esfuerzos de reorganización de la Escuela de Arquitectura de la UNSA. Los principales miembros de esta vanguardia liderada por Edgardo Ramírez (ver Figura 1) fueron Álvaro Pastor, David Quequesana, Alfredo López, Orestes Torres, José Carpio y Carlos Gilt. Entre todos los miembros de esta vanguardia académica arequipeña había una constante: todos habían realizado sus estudios profesionales o estaban estrechamente vinculados con la vida académica argentina. El artículo pretende demostrar esta constante.

\section{El centralismo de las capitales}

La historiografía arquitectónica en países como Perú y Argentina ha estado sometida de una u otra forma a una especie de centralismo geográfico y temático, en virtud del cual los únicos hechos, procesos y personajes reconocidos e historiados eran aquellos producidos en las grandes capitales. Todo lo que ocurría fuera de esos espacios metropolitanos no solo se consideraba carente de valor alguno que legitimase su ingreso a la "historia oficial", sino que terminaba completamente invisibilizado.

Investigaciones recientes y un cambio esencial en el enfoque de la investigación en diversos ámbitos ha permitido revelar lo que siempre se conoció, pero no se aceptaba o reconocía: que muchos de los grandes cambios 
y gestos de vanguardia en el terreno cultural se produjeron fuera de las principales metrópolis, en aquellas regiones remotas o provincias supuestamente alejadas del progreso y la modernidad.

El caso de la extraordinaria y poco conocida contribución de la Facultad de Arquitectura de la UNSA es un ejemplo de los cambios que se produjeron en Perú desde los años 60. Se trata de un fenómeno tan inexplorado como sorprendente en cuanto su conexión con la escena académica argentina.

Conocer este fenómeno y la historia de los vínculos con la experiencia argentina ha sido la principal motivación para abordar este tema. En primer lugar, por haber nacido en Argentina y tener lazos entrañables con el Perú, así como un interés particular en la historia de los vínculos entre ambos países. Y, en segundo lugar, porque me interesa "descentralizar" la historia reconociendo el rol descollante de las provincias en la formación de un proyecto moderno de país, como ya ha ocurrido en Argentina.

A pesar de que Arequipa era una de las pocas provincias que contaban con universidad desde $1714^{1}$, las carreras que se dictaban allí eran básicamente humanísticas. Esto obligaba a todo arequipeño que quisiera estudiar algo diferente a trasladarse a Lima, donde estaban los grandes centros educativos como la Universidad Nacional de Ingeniería (UNI), la Universidad Nacional Mayor de San Marcos (UNMSM) y la Universidad Nacional Federico Villareal (UNFV).

Algunos de los protagonistas de la conexión académica entre Arequipa y Argentina contaron cuando los entrevisté cuál fue su historia, y por qué fueron a estudiar a Lima, pero no se quedaron a terminar sus carreras allí. Orestes Torres comentó que el centralismo de la

1 La primera universidad en Arequipa se fundó el 22 de enero de 1714, en el Convento Santo Domingo. Cinco años después, en 1719, los dominicanos debieron cerrarla por no poder sostenerla. El 11 de noviembre de 1828 se registra el acta de fundación de la Universidad Nacional del Gran Padre San Agustín del departamento de Arequipa, que es la que continúa hasta el día de hoy. capital hacía que los que habitaban en las provincias tuvieran que trasladarse a Lima para estudiar, o al exterior. Entusiasmados para realizar sus estudios universitarios, viajaron a Lima Carlos Gilt, Álvaro Pastor y Alfredo López, entre otros. A pesar de ello la mayoría regresó a Arequipa antes de pasado un año. Alfredo López resumió el porqué de esta decisión: él estudió en la UNI, cuando todavía era la Escuela de Ingenieros y Arquitectos, pero el costo de vivir en Lima era muy alto. Su hermano mayor estudiaba Medicina en la ciudad de La Plata, en Argentina, y gastaba mucho menos; así tomó la decisión de dejar los estudios en Lima y radicarse en La Plata.

La decisión de la mayoría de los arequipeños de trasladarse a Argentina se fundamentaba en que esta les daba la posibilidad de formarse en una prestigiosa universidad estatal con profesionales reconocidos, como en Lima, sin tener que abonar por sus estudios, además de que el costo de vida en cualquier ciudad argentina de provincia era mucho menor que en Lima.

\section{Buscando nuevos horizontes en Argentina}

Ninguno de los jóvenes pertenecientes a la vanguardia académica arequipeña fue a estudiar a Buenos Aires, capital de Argentina, sino que se formaron en diferentes centros regionales del país, como Resistencia, Tucumán, Córdoba y La Plata. La decisión de dónde estudiar respondio a diferentes motivos para cada uno de los integrantes de la vanguardia.

La primera conexión académica se dio cuando Carlos Gilt viajó a Resistencia, donde vivían sus abuelos y uno de sus hermanos. En 1956 había abierto sus puertas la Universidad Nacional del Noreste (UNNE), cuya pequeña Escuela de Arquitectura estaba dirigida por el arquitecto Roberto Champión, quien trabajaba allí junto con sus colegas Mario Molina y Vedia, Juan Molina y Vedia (JMV) (hijo de Mario), Blanca Fabricant y Rodolfo Livingston, todos egresados de la Universidad de Buenos Aires (UBA). 
JMV fue el encargado de organizar el nuevo plan de estudios para la escuela de Arquitectura, el llamado Plan Chaco, el cual buscaba incorporar las inquietudes que se planteaban en ese momento en las distintas escuelas de Arquitectura del país (Córdoba, La Plata, Buenos Aires). Él se acababa de graduar y tenía presentes las discusiones que se daban en las escuelas de Arquitectura sobre cómo debía ser la práctica profesional del arquitecto. Así, JMV propuso en el plan en cuestión "despertar en el estudiante el sentido de responsabilidad histórica que le habilite para controlar la utilización de sus conocimientos a los fines perseguidos" (Coccato, 2011, p. 19). Para lograr este objetivo, pensó que era necesario dar al estudiante universitario una formación con una sólida conciencia histórica de los problemas nacionales.

La metodología del Plan Chaco proponía lo siguiente:

1. Estudiar la arquitectura de una manera diferente: "Se abandona la idea del edificio como un único objeto de estudio, para incorporar, una idea totalizadora dentro de la cual interactúan el medio-físico y, fundamentalmente, lo social: sus habitantes y sus relaciones-, y claro está, los objetos arquitectónicos. Pero de una manera tan dinámica, que, desde entonces, será imposible hablar de arquitectura y no hacerlo en relación a la vida" (Coccato, 2011, p. 11).

2. Enseñar arquitectura de una manera diferente: "tan alejada de la clase magistral y tan cercana al alumno, comprendiendo sus dificultades, guiando su aprendizaje y, fundamentalmente, haciendo crecer la llama con que todo joven ingresa a la universidad, sus potencialidades, sus expectativas" (Coccato, 2011, p. 11).

3. "Promover el debate entre profesores y alumnos sobre los problemas de la enseñanza de la arquitectura, con la intención que el alumno se ubique históricamente en su época" (Coccato, 2011, p. 22), analizando el proceso de desarrollo social de la nación y de la región en la que ejercerá su profesión. De esta manera dejaba atrás al hombre como centro de la preocupación de la Arquitectura, colocando en su lugar el desarrollo de la sociedad en sí misma.

4. Incorporar también el estudio de la "dimensión local, regional, nacional y universal como una parte indispensable del conocimiento" (Coccato, 2011, p. 12) del arquitecto. JMV opinaba que "es indispensable ir más allá de las vagas generalidades, sería la base para la creación de un fructífero espíritu regional, intención que por otra parte es una de las bases que rige la función de nuestra universidad y que ha sido ya concretada en gran parte con el trabajo realizado en nuestra escuela" (Coccato, 2011, p. 24).

Carlos Gilt estudió en la UNNE durante cinco años, pero por motivos personales regresó a Arequipa cuando aún le faltaba un año de estudios para graduarse. Más tarde culminaría la carrera en la Escuela de Arquitectura de la UNSA.

Otro joven estudiante arequipeño, David Quequesana, optó por el Instituto de Arquitectura de Tucumán (IAT); él sabía que esta universidad era el centro de formación por excelencia de la zona norte de Argentina. El presidente argentino Juan Perón, en 1946, había nombrado interventor de la Universidad Nacional de Tucumán ${ }^{2}$ (UNT) a su amigo el Dr. Descole, y le había facilitado todo lo necesario para que cumpliera el objetivo de excelencia de la casa de estudios. En una carta personal que le escribió Perón el 3 de octubre de 1947, le contaba lo siguiente: "Estoy persuadido que usted hará allí una universidad modelo, por eso disfruto de sus triunfos, como amigo y como compatriota. Con el estímulo, que siempre llega al espíritu que trabaja por su intermedio en la palabra sincera de los demás, reciba un abrazo de su amigo. Juan Perón" (Aceñolaza, 2017, p. 453).

El Dr. Descole convocó para organizar la Escuela de Arquitectura a los arquitectos Eduardo Sacriste, Horacio Caminos y Jorge

2 Durante el periodo en que la Universidad Nacional de Tucumán estuvo a cargo del Dr. Descole, la casa de estudio se organizó en institutos, entre ellos el Instituto de Arquitectura. 
Vivanco. Los tres formaban parte del Grupo Austral $^{3}$, dirigido por Kurchan, Ferrari Hardoy y Bonet, seguidores del pensamiento racionalista de Le Corbusier, e impulsadores de la arquitectura moderna y el urbanismo en la Argentina.

El arquitecto Vivanco viajó a Europa para participar del CIAM del año 1947 en Bridgewater, Inglaterra, donde contrató a un grupo de italianos para que los ayudaran en la tarea de organizar y dictar clases en el Instituto de Arquitectura. Los italianos accedieron de inmediato a la propuesta, ya que la situación en Europa era muy difícil -había terminado hace poco la Segunda Guerra Mundial- y, como muchos europeos, buscaban nuevos horizontes fuera del viejo continente. Llegaron así a Tucumán, en 1959, los arquitectos C. Calcaprina ${ }^{4}$, Enrico Tedeschi, L. Picciano y Ernesto Rogers, entre otros.

El nuevo plan de estudios, organizado por Eduardo Sacriste, marcó el rumbo del Instituto de Arquitectura. En este se aplicaba la metodología de organizar los talleres a partir de temas que surgieran de "clientes reales", como lo venía haciendo la Bauhaus. La misión del instituto era educar para transformar al alumno en el generador de hechos arquitectónicos concretos. El plan priorizaba la investigación, proyección y construcción. Sacriste presentó los lineamientos generales de todas las materias, con el objetivo de dar una unidad al plan, cuyo eje central es la Ar-

3 La Agrupación Austral se formó en 1938 en Buenos Aires. Los líderes de la misma -Kurchan, Ferrari Hardoy y Bonet- se habían conocido trabajando en el estudio de Le Corbusier en París, en 1937. Los dos primeros habían llegado a Europa junto con sus compañeros de promoción para realizar el viaje de egresados de la carrera y finalizado el mismo se habían quedado trabajando en el estudio de Le Corbusier. Allí conocieron al catalán Bonet. Los tres se hicieron amigos y decidieron regresar a Buenos Aires llevando consigo los ideales del racionalismo y de la arquitectura moderna. En 1939 publicaron su manifiesto en Buenos Aires y trataron de continuar con las ideas propuestas por Le Corbusier en 1929 para esa ciudad.

4 Todos ellos participantes del grupo Asociación para la Arquitectura Orgánica (APAO), y del movimiento organicista liderado por los teóricos Bruno Zevi y Lewis Munford. La revista Metrón era el órgano de difusión de las ideas de la arquitectura orgánica de la época. quitectura. Él propuso que los conocimientos se adquieran de forma intensiva, que el alumno tenga una participación activa para habituar así a la mente de los mismos a resolver mentalmente los problemas. Siguiendo esos planteamientos, la construcción de la ciudad universitaria sería la "síntesis acabada de aquellos fines didácticos".

La trasformación en el pensamiento, la presencia de personalidades jóvenes nacionales e internacionales con capacidad para vislumbrar y concretar procesos de cambio, y la validación social, con la base teórica del movimiento moderno, hicieron viable la materialización de esta propuesta e implicaron el punto de cambio o inflexión en el Instituto de Arquitectura y Urbanismo de Tucumán (IAUT). Para Longoni, "Lo que 'a priori' podría estimarse como una mezcla potencialmente explosiva: llevar un grupo de militantes 'orgánicos' (los italianos) a un centro hegemonizado por 'lecorbusieranos' (Sacriste, Caminos y Vivianco), resultó altamente positiva" (Longoni \& Fonseca, s.f., p. 6).

Tedeschi se desempeñó como titular de Historia de la Arquitectura (HA) y Teoría de la Arquitectura (TA), y publicó libros sobre el tema especialmente para ser usados en el IAT $^{5}$. Organizó junto con Francisco Bullrich y Marina Waisman el Instituto de Historia de la Arquitectura y del Arte, que funcionó simultáneamente en las universidades de Tucumán, Córdoba y Cuyo, y que luego, en 1972, se incorporaría la Universidad de Mendoza, entonces dirigida por Tedeschi. Como aseverara Liernur, Tedeschi dejó ejemplificadas las ideas que difundía:

Ha dejado diversas obras donde prioriza el manejo depurado de los elementos constructivos, el rechazo de las modas transitorias y el ajuste de soluciones a las condiciones regionales, especialmente las climáticas y tecnológicas. Así lo demuestran varias casas, hoteles y mercados, destacándose la Universidad de Mendoza. (Liernur \& Aliata, 2004, p. 107)

El Instituto de Arquitectura y Urbanismo de Tucumán pasó a ser solo Instituto de Arquitectura de Tucumán (IAT). 
Al final de su primer año de estudios en la Universidad Nacional de Tucumán (UNT), David Quequesana retornó a Arequipa durante sus vacaciones, donde se encontró con Álvaro Pastor, quien regresaba decepcionado de su experiencia en Lima. Quequesana animó a su amigo a que lo acompañase a estudiar en Tucumán, donde él había tenido una muy grata experiencia. Así, al finalizar el verano, ambos partieron hacia Argentina. En Tucumán trascurren los años de facultad compartiendo amigos y estudios. Entre sus compañeros mayores estaba el hoy conocido arquitecto Cesar Pelli. Años después ambos regresarían graduados de arquitectos a su ciudad natal y se incorporarían al cuerpo de profesores de la UNSA.

Una nueva conexión académica se estableció a principios de los años 60, cuando Edgardo Ramírez Chirino también viaja a estudiar en Argentina, en su caso a Córdoba, a la Escuela de Arquitectura de la Universidad Nacional de Córdoba (UNC).

Si bien es cierto que el IAT fue pionero en cuanto a las nuevas propuestas de enseñanza de Arquitectura, esta renovación también ocurrió en otras casas de altos estudios de Argentina, como es el caso de la UNC, creada en 1954. La nueva Facultad de Arquitectura y Urbanismo (FAU), como todas las fundadas en esta época, comenzó orientada bajo la metodología de L'École des Beaux-Arts. Sin embargo, un pequeño grupo de docentes propuso desde sus orígenes el acercamiento a la arquitectura moderna. Entre ellos podemos nombrar a Ernesto La Padula, Marina Waisman, Jaime Roca y Julio Pinzani. La Padula era un reconocido arquitecto urbanista italiano, que había llegado con la camada de italianos radicados en Tucumán entre 1958 y 1959, y participaba junto con ellos del compromiso con la arquitectura moderna. La Padula era miembro del Movimiento Italiano de Arquitectura Racional (MIR), ingresó a trabajar en el equipo técnico del plan regulador de Córdoba, y en la UNC se hizo cargo del Taller de Composición IV (tesis) y el de Urbanismo: "Como docente, La Padula, produjo importantes cambios en la enseñanza al unificar los propósitos de la arquitectura y el urbanismo" (Longoni \& Fonseca, s.f., p. 23).

Hacia finales de la década del 60 , los conflictos políticos que vivía la Argentina habían generado la politización de las cátedras de la UNC. En este clima de crecientes enfrentamientos, el decano Rogelio Luque puso en funcionamiento el Consejo Académico -contemplado en la ley universitaria $\mathrm{N}^{\circ} 17.245$ de 1967-, en un intento infructuoso por controlar la situación que se vivía en la facultad. Marina Waisman ${ }^{6}$ fue elegida vicedecana en abril de ese año; y Enrico Tedeschi, nombrado parte del equipo director que debía poner en funcionamiento los llamados "talleres verticales"(Malecki, 2016).

Los arquitectos Jaime Roca y Enrico Tedeschi incorporaron dentro de los cambios pedagógicos algunas ideas que ya se venían implementando en el Instituto Interuniversitario de Historia de la Arquitectura (IIDEHA), entre ellas la elaboración de publicaciones que ayudaran a propulsar el desarrollo de la crítica de arquitectura en el país, incorporando al pensamiento local las ideas de las personalidades de la arquitectura de la época. Visitaron el país durante ese período Nikolaus Pevsner, Giulio Argán, Fernando Chueca y Goitia, Joshua Taylor, Guideon, Zevi y Benévolo.

Para finalizar sus estudios, Edgardo Ramírez Chirino realizó una tesis titulada Renovación Urbana de Arequipa, Perú, bajo la dirección de los arquitectos Enrico Tedeschi y Miguel Ángel Roca. En el examen de su tesis obtuvo la calificación máxima que otorga la universidad de Argentina, 10 puntos, sobresaliente, por el Tribunal Examinador (certificado emitido por la UNC, firmado por el Arq. Marcelo Novillo Corvalán).

Edgardo Ramírez compartió una gran amistad con Miguel Ángel Roca hasta el día de su muerte. Roca lo visitó varias veces en Arequipa y dictó seminarios en la UNSA. Ramírez, una vez graduado, regresó a Arequipa

6 Marina Waisman se había graduado como arquitecta en la UNC en 1945. En 1947 fue nombrada adjunta de Historia de la Arquitectura II en la misma universidad. Participaba junto con Tedeschi y Jaime Roca del IIDEHA. 
y, al igual que David Quequesana y Álvaro Pastor, comenzó a trabajar en la UNSA como profesor, donde propuso grandes cambios y la introducción de metodologías aprendidas en Córdoba.

Un nuevo vínculo se estableció gracias a que Alfredo López, Oreste Torres y Lucho Alarcón partieron a estudiar a la Universidad de La Plata (UNLP), a solo 60 kilómetros de la capital argentina. Ellos consideraron una ventaja el hecho de que La Plata es una ciudad principalmente estudiantil, donde concurrían alumnos de toda Argentina y de los países cercanos. Esto les otorgaba la ventaja de poder formarse con profesionales reconocidos a nivel nacional que por la cercanía a Buenos Aires enseñaban en ambas facultades (UNLP y UBA).

La carrera de Arquitectura y Urbanismo en la UNLP había sido creada en 1951. Su vicedecano, el ingeniero Arturo Guzmán, había sido antes decano en la UNT. Gracias a Guzmán, en 1952 comenzaron a llegar a Buenos Aires algunos arquitectos que habían trabajado en el IAT, pero tras la renuncia del Dr. Descole como interventor del Instituto, también habían dado un paso al costado. En 1953 se incorporaron al grupo de trabajo en La Plata los arquitectos Vivanco, Díaz Puertas, Borgatto, Hilario Zalba y el italiano Rafael Onetto.

En el primer plan de estudios de Arquitectura de la UNLP, propuesto en 1953, el Taller de Arquitectura Vertical estaba considerado la materia central de la carrera. Además, cursos como Historia y Teoría de la Arquitectura del Urbanismo ganaron relevancia dentro del plan respecto de las materias exactas, que siguieron siendo dictadas por ingenieros. La metodología aplicada era muy similar a la que se había propuesto algunos años antes en Tucumán, Argentina.

López, Torres y Alarcón también se graduaron de arquitectos urbanistas y regresaron a Arequipa a unirse con la vanguardia académica arequipeña, para reformar juntos la metodología y el plan de estudios de la Escuela de Arquitectura de la UNSA.

\section{La sucursal de Lima (1964-1966)}

En la década del 60 proliferaron las universidades en el Perú: se inauguraron en Huancayo, Arequipa, Cerro de Pasco, Cajamarca, Ayacucho, Ica, Iquitos, Huánuco, Puno, Huacho y Lima (Sumi, 2014).

Arequipa, más que cualquier otra provincia, "requería formar arquitectos competentes que fueran conocedores del tema y que no pudieran hacer distorsiones del legado arquitectónico de nuestro patrimonio monumental" (Alpaca, 2015, p. 6), pues atravesaba una etapa de reconstrucción de su provincia luego de los terremotos de 1958 y 1960. Era imperante asegurar la reconstrucción con suficiente cuidado y conciencia ciudadana. En Perú en general se

empezará a reconocer el pequeño o gran aporte de la universidad al desarrollo del país, implica un cambio de visión y de actitud frente al problema. Implica, entre otras cosas, reconocer que el valor y la importancia, de una experiencia nuestra lo fijamos nosotros mismos en función no solo de las urgencias y posibilidades de desarrollo de nuestro propio país, sino también de la capacidad de la universidad para contribuir a la solución de sus problemas específicos (Ludeña, 1990, p. 12).

En 1964 se creó la Escuela de Arquitectura de la UNSA, tal como lo confirma el Acta de Sesión de la Escuela Profesional de Arquitectura de fecha 6 de junio de 1964, en el inciso D:

Se acordó así mismo agradecer a la Facultad de Arquitectura de la Universidad Nacional de Ingeniería, por la colaboración que ha prestado para la creación y funcionamiento de la Escuela Profesional de Arquitectura de la Universidad Nacional San Agustín, haciendo extensivo este agradecimiento al Arquitecto Santiago Agurto. (UNSA, Actas de Sesiones, 1964 , p. 4)

Entre todas las nuevas facultades, el caso de Arequipa resulta especial, no solo por su conexión con la escena académica profesional argentina, sino por la resonancia pública y el activismo nacional fomentado por los arquitectos que gestaron los cambios alrededor de las décadas de 1960 y 1970. 
Arequipa se encontraba en proceso de reconstrucción, para lo cual era necesario capacitar arquitectos competentes que no "distorsionaran" el legado del patrimonio de la ciudad. El objetivo era alcanzar "una identidad del pensamiento y la práctica arquitectural peruana y latinoamericana, una relación más estrecha entre la enseñanza y las necesidades reales de la sociedad peruana, el uso de nuevas tecnologías apropiadas al medio, entre otras demandas" (Ludeña, 1990, p. 20).

En un principio, la Escuela de Arquitectura de Arequipa contó con la colaboración de la UNI; esta les brindó los planes de estudio, su metodología y envió profesores e incluso se organizaron viajes de estudio a Lima. Así lo expresa el Acta de Sesión de la Escuela, donde se indica que se utilizarán "todos los programas de la Facultad de Arquitectura de Lima" (UNSA, Actas de Sesiones, 1964, p. 9).

En Lima, la Agrupación Espacio en 1947 publicó un manifiesto donde repudiaba el historicismo académico proveniente del Viejo Continente y reclamaba que la orientación que se daba a la carrera no respondía a las necesidades de la época. Los nuevos objetivos buscaban que la educación se alineara en dirección a la propuesta de Walter Gropius y la escuela Bauhaus. La propuesta de la Agrupación Espacio se asemejaba a la realizada por la Agrupación Austral en Argentina en 1939.

En Arequipa no solo se replicaban las demandas de Lima que demandaban la modernidad, sino que además se reclamaba la falta de un modelo pedagógico y se manifestaba el descontento por la insuficiencia de profesores arquitectos que llegaban de la UNI de Lima a dictar clases a Arequipa. Según la asistencia de la sesión del 9 de julio de 1964, solo había dos arquitectos (Alberto Araenzaenz y Hugo Ruiz de Samocurcio), mientras que se contaba con siete ingenieros (Alfonso Delgado Lira, Lupe Arlot Tamayo Ballón, Victor Oviedo, Daniel Ochoa Miranda, José Vela Escobedo, Nicanor Hurtado de Mendoza y Edgardo Zamallo Zapata).

Fueron muchos los problemas que se generaron entre la UNI y la UNSA. La aceptación de la UNSA de dejar ingresar a todos los postulantes inscritos a la Escuela de Arquitectura fue el conflicto que marcó el fin de la relación. La UNI decidió entonces retirar su apoyo. Sin embargo, como evidencia del espíritu audaz y emprendedor de los arequipeños, ante el "abandono" súbito de la UNI, en vez de abatirse o empezar una larga demanda dirigida a Lima, las autoridades de la UNSA decidieron aprovechar estas circunstancias dramáticas para dar un salto al futuro. Entonces la Escuela de Arquitectura de Arequipa se declaró autónoma, según lo señala el Acta de Instalación del 23 de julio de 1966:

El señor profesor Eduardo Ugarte y Ugarte hizo uso de la palabra quien a nombre propio y de los miembros de la Asamblea y de los alumnos componentes del Tercio estudiantil, con las debidas consideraciones y apreciación del acto de tanta trascendencia para la Escuela y la Universidad de haberse logrado la autonomía de la Escuela de Profesionales de Arquitectura, pidió un voto de aplauso y de agradecimiento por la feliz gestión en la persona del ingeniero Delgado Lira, director interino de la Escuela (UNSA, Actas de Sesiones, 1966-67, p. 2)

\section{La autonomía de la Escuela de Arquitectura} de la UNSA. Llegan los docentes argentinos (1966-1967)

La ausencia de profesores preparados adecuadamente en Arequipa llevó al ingeniero Alfonso Delgado, director interino de la Escuela de Arquitectura de la UNSA, a contratar a docentes capacitados para cumplir con las exigencias de una nueva enseñanza de la Arquitectura. Carlos Gilt acababa de regresar a Arequipa y propuso al ingeniero Delgado contactarse con los profesores de la Escuela de Arquitectura de la UNNE de Argentina. Luego de un par de meses de tratativas, en 1967 llegó a Arequipa una nueva conexión académica, esta vez en el otro sentido, de Argentina a Arequipa, representada por los arquitectos Juan Molina y Vedia, Blanca Fabricant y Rodolfo Livingston (ver Figura 2).

El ingeniero Delgado les solicitó a los profesionales argentinos que lo ayuden a reestructurar el plan de estudios y la metodología según las necesidades de Arequipa. Estos 
propusieron adoptar la metodología del Plan Chaco, utilizado en la UNNE, y adaptarlo a las necesidades y a la realidad arequipeña. Se hicieron cargo de los talleres de Arquitectura, y de las cátedras de Historia y de Representación. La biblioteca de la universidad era escueta, por lo que los argentinos aportaron su biblioteca personal y solicitaron a la escuela que se compraran más libros. Llegaron así a la biblioteca de la UNSA libros de teóricos y críticos argentinos como Marina Waisman y Francisco Bullrich, y del italiano Enrico Tedeschi, junto con revistas especializadas en la disciplina.

Coccato -alumno de los arquitectos argentinos en la UNNE- expresó sobre ellos: "Estos arquitectos propusieron una manera de enseñar, tan alejada de la clase magistral y tan cercana al alumno, comprendiendo sus dificultades, guiando su aprendizaje y fundamentalmente, haciendo crecer la llama con que todo joven ingresa a la universidad, sus potenciales, sus expectativas" (2011, p. 11). Según Marizela Alpaca, fueron los

Arq. Juan Molina y Vedia, el Arq. Rodolfo Livingston y la Arq. Blanca Fabricant de Molina, quienes permanecieron en la ciudad de Arequipa por el lapso de un año, quienes marcaron un cambio en el sistema pedagógico de la escuela, dándole un enfoque más arquitectónico y dejando la visión ingenieril que se manejó en los tres años anteriores, mientras la escuela estuvo bajo la dependencia de la Facultad de Arquitectura de la UNI. (2013, p. 7)

Lamentablemente, por problemas de convalidación de los títulos y por la legislación existente en ese momento en el Perú, no se pudo renovar el contrato con los argentinos. Al finalizar el año lectivo de 1967 regresaron a su país de origen. Nuevamente la Escuela de Arquitectura de Arequipa se encontraba sin docentes. Si bien las bases del sistema pedagógico fueron trasmitidas inicialmente durante el breve año en el que estuvieron estos arquitectos argentinos, se considera que los que fortalecieron sus cimientos pedagógicos y la trasmisión del plan de estudios utilizado en Argentina fueron los integrantes de la vanguardia académica arequipeña liderada por Ramírez Chirino, con quien trabajaron

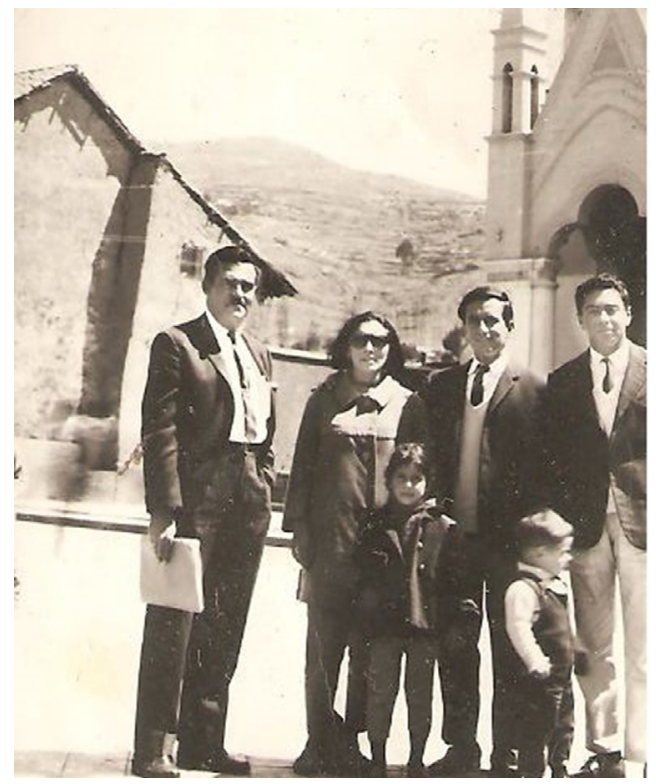

Figura 2. Los argentinos Juan Molina y Vedia, Blanca Fabricant y Rodolfo Livingston en Puno junto con el arquitecto Araenzaenz. L. Molina y Vedia, 1967.

Álvaro Pastor, David Quequesana, Alfredo López, Orestes Torres y José Carpio.

\section{La vanguardia académica arequipeña (1968- 1987)}

Durante este periodo la vanguardia académica arequipeña se incorporó en la organización de la Escuela de Arquitectura, utilizando los criterios pedagógicos aprendidos en Argentina, modificando los planes de estudio y la metodología de enseñanza; de tal manera se involucraron, que cuando fue elegido el gobierno tripartito de la carrera el arquitecto Edgardo Ramírez Chirino fue designado presidente. Ramírez logró el sueño de la Escuela de proyectar la ciudad Universitaria de la Av. Venezuela, y trasladar la Escuela de Arquitectura allí.

Los primeros cambios metodológicos realizados en la Escuela de Arquitectura, como ya se ha mencionado, fueron realizados por los argentinos, que adaptaron y aplicaron el 
Plan Chaco. Continuaron con esa labor los arquitectos José Carpio Núñez, Sarmiento Pinto y Málaga González con la redacción del llamado documento básico, el cual "tiene por objetivo, establecer, los lineamientos a partir de los cuales se desarrollarán las acciones del programa Académico, orientadas a conseguir una reconceptualización del hecho arquitectónico y su enseñanza" (Carpio Núñez, Sarmiento Pinto \& Málaga González, 1972, p. 3). En este documento, los exalumnos de los arquitectos argentinos tomaron elementos desarrollados por MYV en el Plan Chaco y enfatizaron la orientación social a la que se quería llevar a la Facultad de Arquitectura de la UNSA, consecuente con los cambios y modificaciones de la época.

Luego siguieron adelante con los cambios los integrantes de la vanguardia, que aplicaron los criterios pedagógicos y las metodologías que ellos habían conocido en Argentina. En 1974 estos cambios pedagógicos comenzaron a hacerse conocidos en el Perú, a raíz del encuentro en Huancayo de las Facultades de Arequipa, donde los representantes de la UNSA se destacaron -como se comentó en el inicio del artículo- por el nuevo enfoque en los proyectos, metodologías y planes de estudio.

En 1977 se cambió nuevamente el plan de estudios de la carrera de Arquitectura de la UNSA, pero definitivamente el punto que denotó claramente la conexión entre los dos países fue cuando el 27 de marzo de 1978 el ingeniero Jorge Vargas Calas, director del programa académico de Arquitectura de la UNSA, presentó al Sr. Dr. José Gutiérrez Correa, rector de la universidad. El Plan de Reorganización del Programa Académico de Arquitectura, en el segundo párrafo de la presentación, expresaba claramente tal vínculo y similitud en la enseñanza:

El trabajo de reordenamiento presentado deja ver claramente que se ha adoptado una estructura general muy similar, por no decir igual a la estructura de las Facultades de Arquitectura de la Nación Argentina, que, desde luego, son homogéneas y casi perfectamente concebidas. (Vargas Calas, 1978, p. 1)
Ya en 1984, se comenzó a entregar el título de "arquitecto y urbanista". En 1987, cuando el arquitecto Edgardo Ramírez fue nombrado decano de la UNSA, organizó junto con José Carpio los talleres verticales, basándose también en la experiencia previa que había acumulado personalmente asistiendo a talleres en Córdoba.

En lo referente a la construcción, al poco tiempo de regresar a Arequipa, los arquitectos Ramírez, Pastor y Carpio ganaron un concurso de la Mutual. La propuesta arquitectónica para este proyecto era muy diferente a lo que se venía proponiendo en la arquitectura de Arequipa de los años 70; sin embargo, se podía hallar en ella una cierta relación con la arquitectura regional que venían proponiendo en Argentina los arquitectos Roca, Tedeschi y Sacriste. En los años siguientes Álvaro Pastor fue conocido por la construcción de la Posada del Puente, el Pinar del Río y los Hoteles construidos en el Colca, entre otras obras.

\section{Conclusiones}

El centralismo predominante en el Perú de los años 60 llevó a muchos jóvenes a alejarse de sus hogares para poder formarse en una profesión. Argentina, que contaba con diferentes universidades nacionales en sus provincias, hizo posible que estos jóvenes se formaran en ellas, en lugares donde el costo de vida era menor que en Lima.

Las universidades argentinas contaban con gran cantidad de profesores extranjeros, especialmente las facultades de Arquitectura, que contaban con docentes italianos que trajeron consigo nuevos criterios pedagógicos. La Arquitectura se estudiaba en talleres, a partir de temas que surgieran de clientes reales, como lo venía haciendo la Bauhaus. Aprendiendo a investigar, proyectar y construir, teniendo en cuenta el contexto regional, climático y social, se promovía el debate entre profesores y alumnos, para que estos aprendieran a justificar y razonar sus propuestas.

Los arequipeños aprovecharon todo el conocimiento al que allí tuvieron acceso, se graduaron en estas universidades argentinas y 
regresaron a su ciudad de origen, donde conformaron una vanguardia académica. Estos se organizaron en pos de cumplir un nuevo objetivo, sacar adelante la nueva Escuela de Arquitectura de Arequipa. Compartieron conocimientos y metodologías, y discutieron diferentes criterios pedagógicos. Finalmente, lograron su objetivo: la Facultad de Arquitectura de la UNSA comenzó a ser conocida en el país por sus nuevos criterios pedagógicos y por las propuestas arquitectónicas que construían los integrantes que conformaban la vanguardia académica arequipeña.

\section{Referencias}

Alpaca, M. (2015). Historia de la Facultad de Arquitectura y Urbanismo de la Universidad Nacional San Agustín. Revista de Arquitectura. Urbanismo y Territorio, año 1, 6-12.

Aceñolaza, F. (2017). La Universidad de Tucumán en la década 1945-1955. Recuperado de http://www.archivo.unt.edu.ar/attachments/059_ ace\%C3\%B1olaza.pdf

Carpio Nuñez, J., Sarmiento Pinto, D. \& Málaga González, F. (1972). Programa de Arquitectura-Documento básico. Arequipa, Perú: Universidad Nacional San Agustín de Arequipa.

Coccato, M. (2011). El Plan Chaco. Juan Molina y Vedia. 1959. Corrientes, Argentina: Universidad Nacional del Noreste.

Liernur, F. \& Aliata, F. (2004). Diccionario de Arquitectura en la Argentina. Buenos Aires, Argentina: Artes Gráficas Rioplatenses.

Longoni, R., \& Fonseca, I. (s.f.). La enseñanza de la Arquitectura y el Urbanismo en el primer gobierno peronista. Recuperado de $2^{\text {a }}$ Congreso Red de Estudios sobre el Peronismo: http://redesperonismo.com. $\mathrm{ar} / \mathrm{archivos} / \mathrm{CD} 2 /$ Longoni.pdf
El aporte realizado por esta vanguardia tuvo fuerte influencia en los arquitectos locales, quienes demostraron siempre tener un marcado respeto por el hombre, su historia y su contexto, y propusieron proyectos que partían de un claro análisis de la persona, el entorno y las características del lugar. Ejemplo de ello son las obras que realizaron, como la Mutual y la Posada del Puente. Hoy algunos de los integrantes, como Álvaro Pastor, continúan enseñando en la UNSA y sus alumnos mantienen ese respeto hacia el hombre, su historia y su contexto.

Ludeña, W. (1990). De la u-topía a la a-patía. Reflexiones acerca de un itinerario académico en arquitectura. Lima, Perú: Arius.

Malecki, J. S. (2016). Crisis, radicalización y politica en el Taller Total de Córdoba, 1970-1975. Prohistoria, 25, 79-103. Recuperado de http://www.academia. edu/4237242/Historia_y_cr\%C3\%ADtica._Enrico_ Tedeschi_en_la_renovaci\%C3\%B3n_de_la_cultura_ arquitect $\%$ C $3 \% \bar{B} 3$ nica_argentina_1950-1970

Sumi,A.(4demayo, 2014).Los Andes. Recuperadodehttp:/ / www.losandes.com.pe/Educacion/20140504/79956.html

Universidad Nacional San Agustín de Arequipa., C. d. (1964). Actas de Sesiones. Arequipa.

Universidad Nacional San Agustín de Arequipa., C. d. (1966-67). Actas de Sesiones. Arequipa.

Vargas Calas, J. (1978). Plan de reorganización del programa académico de arquitectura. "Plan A". Arequipa, Perú: Universidad Nacional San Agustín de Arequipa. 